\title{
LONG-TERM OPTICAL OBSERVATIONS OF TWO LMXBs: UW CrB (=MS 1603+260) AND V1408 Aql (=4U 1957+115)
}

\author{
Paul A. Mason ${ }^{1,2}$, Edward L. Robinson ${ }^{3}$, Amanda J. Bayless ${ }^{3,4}$, And Pasi J. Hakala ${ }^{5}$ \\ ${ }^{1}$ Department of Physics, University of Texas at El Paso, El Paso, TX 79968, USA \\ 2 Department of Mathematics and Physical Sciences, New Mexico State University-DACC, Las Cruces, NM 88003, USA \\ ${ }^{3}$ Department of Astronomy, University of Texas at Austin, 1 University Station, Austin, TX 78712, USA \\ ${ }^{4}$ Southwest Research Institute, 6220 Culebra Road, San Antonio, TX 78238, USA \\ ${ }^{5}$ Finnish Centre for Astronomy with ESO, Väisäläntie 20, FIN-21500 Piikkiö, University of Turku, Finland \\ Received 2011 December 3; accepted 2012 July 24; published 2012 September 11
}

\begin{abstract}
We present new optical photometry of two low-mass X-ray binary stars, UW CrB (MS 1603+260) and V1408 Aql (4U 1957+115). UW CrB is an eclipsing binary and we refine its eclipse ephemeris and measure an upper limit to the rate of change of its orbital period, $|\dot{P}|<4.2 \times 10^{-11}$ (unitless). The light curve of UW CrB shows optical counterparts of type I X-ray bursts. We tabulate the times, orbital phases, and fluences of 33 bursts and show that the optical flux in the bursts comes primarily from the accretion disk, not from the secondary star. The new observations are consistent with a model in which the accretion disk in UW CrB is asymmetric and precesses in the prograde direction with a period of $\sim 5.5$ days. The light curve of V1408 Aql has a low-amplitude modulation at its $9.33 \mathrm{hr}$ orbital period. The modulation remained a nearly pure sine curve in the new data as it was in 1984 and 2008 , but its mean amplitude was lower, $18 \%$ against $23 \%$ in the earlier data. A model in which the orbital modulation is caused by the varying aspect of the heated face of the secondary star continues to give an excellent fit to the light curve. We derive a much improved orbital ephemeris for the system.
\end{abstract}

Key words: stars: individual (MS 1603+260, 4U 1957+115) - X-rays: binaries

Online-only material: color figure

\section{INTRODUCTION}

Low-mass X-ray binary (LMXB) stars consist of a low-mass secondary star orbiting a neutron star (NS) or black hole primary and transferring mass to the primary via an accretion disk. Their $\mathrm{X}$-ray luminosities are typically dominated by the accretion disk and, if present, the NS, while their optical luminosities are usually dominated by the accretion disk and secondary star. The orbital period, $P$, and the rate of change of the orbital period, $\dot{P}$, are crucial properties of an LMXB. The orbital period is a required ingredient for precise mass determinations. Since the secondary star must fill its Roche lobe, its mean density, $\rho$, is given by $\rho \propto P^{-2}$ (Warner 1995); and since the density of a star is determined by its structure, the orbital period sets the structure of the secondary and then the size scale of the entire system. The mean value of $\dot{P}$ measures the rate at which the binary system evolves and, because $\dot{P}$ responds to mass transfer and mass loss, it strongly constrains models for these processes.

In previous work, we measured the orbital period of the LMXB UW CrB from eclipses in its optical light curve, and we confirmed and refined the orbital period of the LMXB V1408 Aql from a nearly sinusoidal orbital modulation of its optical light curve (Mason et al. 2008; Bayless et al. 2011). The period determinations required unusually large amounts of data, UW CrB because the light curve is multi-periodic and its eclipses are highly irregular and V1408 Aql because the orbital modulation is distorted and often obscured by other random variations in its light curve. As a result, the rate of change of the orbital period of UW CrB has a poor limit, and the orbital ephemeris for V1408 Aql is valid for only the one season we observed it, and is not accurate enough to bridge one-year gaps between observing seasons.

In this paper, we present new optical photometry of both systems. After a brief discussion of the new observations, we devote a section to each star. Combining the new data on UW CrB with data obtained over the last 20 years, we refine its eclipse ephemeris and derive an upper limit to the rate of change of its orbital period. The light curve of UW CrB shows optical counterparts of type I X-ray bursts. We show that most of the optical flux in the bursts comes from the accretion disk, not the secondary star. We find that the mean orbital light curve of V1408 Aql remains a low-amplitude sine curve as it was in the data obtained by Thorstensen (1987) and Bayless et al. (2011). We measure the orbital period of V1408 Aql well enough to merge data from successive observing seasons, allowing us to derive a much more accurate orbital ephemeris. The new data are consistent with models we have previously proposed for both systems (Mason et al. 2008; Bayless et al. 2011).

\section{NEW OBSERVATIONS}

We obtained new optical photometry of UW $\mathrm{CrB}$ and V1408 Aql with the Argos high-speed CCD photometer mounted on the $2.1 \mathrm{~m}$ Otto Struve telescope at McDonald Observatory (Nather \& Mukadam 2004). All the observations were made through a broad " $B V R$ " filter that transmits light from 4130 to $7385 \AA$. The data consist of sequences of CCD images exposed for $10 \mathrm{~s}$ with typical total durations of 2-4 hr per night. We observed UW CrB on five successive nights in 2008 May/June and on six successive nights in 2009 June, accumulating $27.5 \mathrm{hr}$ of photometry (see Table 1); and we observed V1408 Aql for four nights in 2009 June and then again for six nights in 2009 September accumulating $27.6 \mathrm{hr}$ of photometry.

The UW CrB data were reduced using IRAF, ${ }^{6}$ while the V1408 Aql data were reduced using a combination of IRAF

\footnotetext{
6 IRAF is distributed by the National Optical Astronomy Observatory, which is operated by the Association of Universities for Research in Astronomy, Inc., under the cooperative agreement with the National Science Foundation.
} 


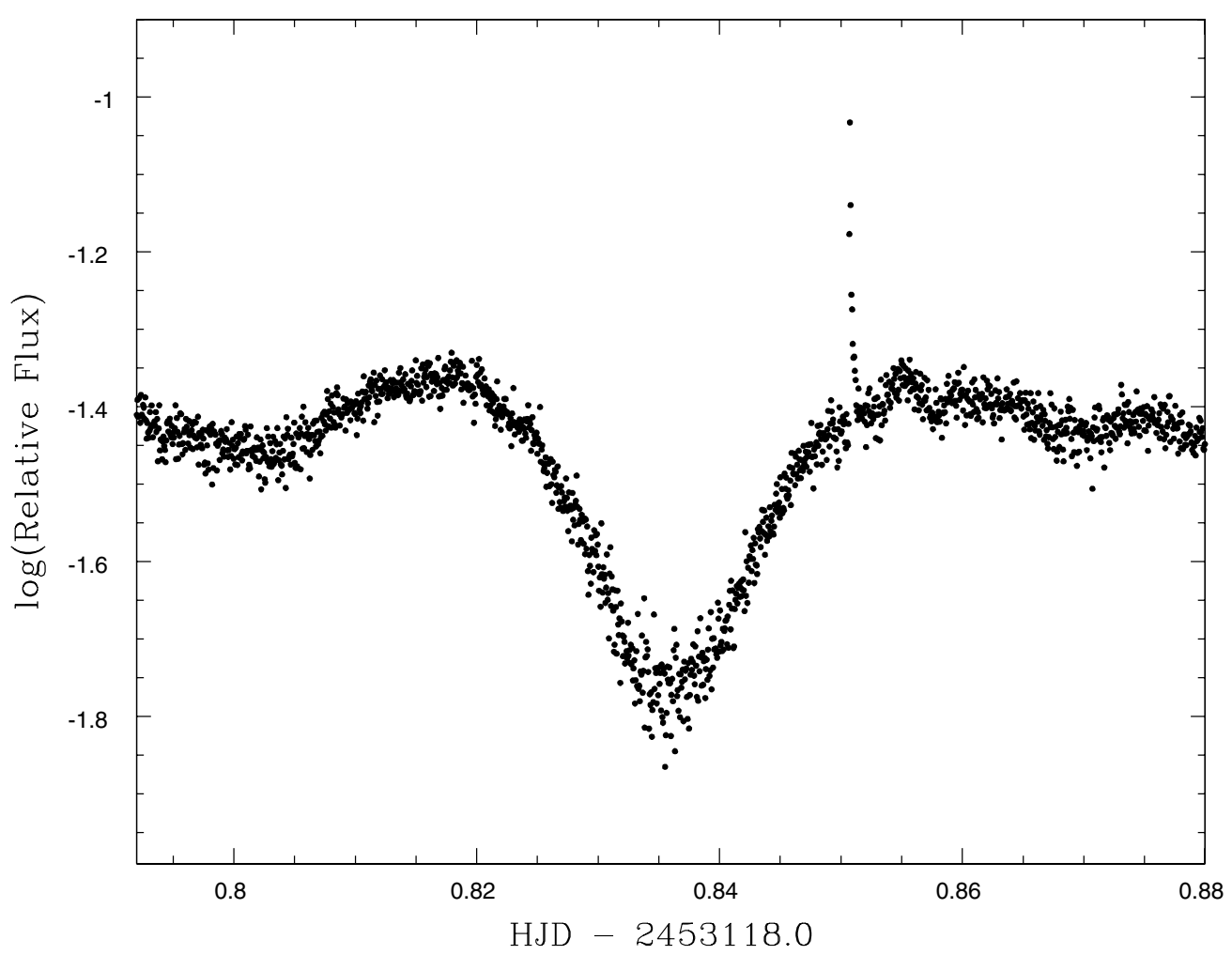

Figure 1. Eclipse of UW CrB on 2004 April 23. The eclipse is also shown in Hynes et al. (2004; the middle eclipse in the bottom light curve of their Figure 1), but here the original $5 \mathrm{~s}$ time resolution has been retained. Note the optical burst just after the end of the eclipse. The burst rose to maximum in less than $10 \mathrm{~s}$ and then faded over the next $60 \mathrm{~s}$.

routines and an IDL pipeline developed by R. Hynes. Additional details about our data reduction methods for UW CrB can be found in Mason et al. (2008) and in Bayless et al. (2011) for V1408 Aql. Sample light curves were reduced by both methods and yielded essentially identical results.

\section{UW CRB}

\subsection{Background}

MS1603+260 was discovered during the Einstein Observatory Extended Medium Sensitivity Survey (Gioia et al. 1990). The X-ray light curve is strongly modulated at a period near $111 \mathrm{~m}$, but there are no obvious eclipses and the morphology of the modulation varies greatly on timescales of two days or less (Hakala et al. 1999; Mukai et al. 2001; Jonker et al. 2003; Hakala et al. 2005). The light curve shows type I bursts, demonstrating that the accreting object is a NS (Mukai et al. 2001; Hakala et al. 2005). Jonker et al. (2003) modeled the X-ray spectral energy distribution with a power law and concluded that MS1603+260 is an accretion disk corona (ADC) system at a distance of 11-24 kpc.

UW CrB, the optical counterpart of MS1603+2600, was identified by Morris et al. (1990). The optical light curve shows four distinct kinds of variability. First, there are seemingly random variations with amplitudes up to $\sim 20 \%$ on timescales of a few minutes and up to a half magnitude on timescales of a few days. These variations overlie and tend to obscure the other variations. Second, there are broad eclipses. The eclipses are often deep and symmetrical as in Figure 1, but the shape and depth of the eclipses vary greatly; furthermore, the eclipse can nearly disappear as shown in Figure 2 and in Figure 1 of Hynes et al. (2004). The orbital phase of mid-eclipse also wanders by up to $\Delta \phi= \pm 0.08$. These are clearly partial eclipses of the
Table 1

Journal of Observations

\begin{tabular}{|c|c|c|c|c|}
\hline \multirow[t]{2}{*}{ UT Date } & \multicolumn{2}{|c|}{ UW CrB } & \multicolumn{2}{|c|}{ V1408 Aql } \\
\hline & UTC Start & $\begin{array}{l}\text { Duration }^{\mathrm{a}} \\
\text { (hr) }\end{array}$ & UTC Start & $\begin{array}{c}\text { Duration }^{\mathrm{a}} \\
\text { (hr) }\end{array}$ \\
\hline 2008 May 30 & 04:05 & 3.2 & $\ldots$ & $\ldots$ \\
\hline 2008 May 31 & $05: 15$ & 2.2 & $\cdots$ & $\ldots$ \\
\hline 2008 Jun 1 & 03:19 & 4.0 & $\ldots$ & $\ldots$ \\
\hline 2008 Jun 2 & 03:03 & 2.0 & $\ldots$ & $\ldots$ \\
\hline 2008 Jun 3 & 03:08 & 3.1 & $\cdots$ & $\cdots$ \\
\hline 2009 Jun 23 & 04:05 & 2.4 & $\ldots$ & $\ldots$ \\
\hline 2009 Jun 24 & $05: 15$ & 2.2 & $07: 44$ & 3.0 \\
\hline 2009 Jun 25 & 03:19 & 2.3 & 06:00 & 2.8 \\
\hline 2009 Jun 26 & 03:03 & 2.1 & $06: 33$ & 4.1 \\
\hline 2009 Jun 27 & 03:09 & 2.1 & $05: 37$ & 2.8 \\
\hline 2009 Jun 28 & $03: 22$ & 1.9 & $\ldots$ & $\ldots$ \\
\hline 2009 Sep 19 & $\ldots$ & $\ldots$ & $02: 30$ & 2.8 \\
\hline 2009 Sep 20 & $\ldots$ & $\ldots$ & 02:02 & 2.4 \\
\hline 2009 Sep 21 & $\cdots$ & $\ldots$ & $01: 53$ & 2.8 \\
\hline 2009 Sep 22 & $\ldots$ & $\ldots$ & 02:05 & 1.4 \\
\hline 2009 Sep 23 & $\ldots$ & $\ldots$ & $02: 31$ & 3.2 \\
\hline 2009 Sep 24 & $\cdots$ & $\cdots$ & $02: 21$ & 2.3 \\
\hline
\end{tabular}

Note. ${ }^{\text {a }}$ Time resolution of all data is $10 \mathrm{~s}$.

accretion disk, not of a solid body. Mason et al. (2008) derived an orbital period of $110.976722 \pm 0.000012$ minutes from the eclipses, but were unable to place a meaningful limit on the rate of change of the orbital period.

Third, the non-eclipse phases of the light curve have a largeamplitude modulation with a period of 112.58 minutes (Mason et al. 2008) or possibly 112.75 minutes (Hakala et al. 2009), about $1.5 \%$ longer than the orbital period. This modulation is 


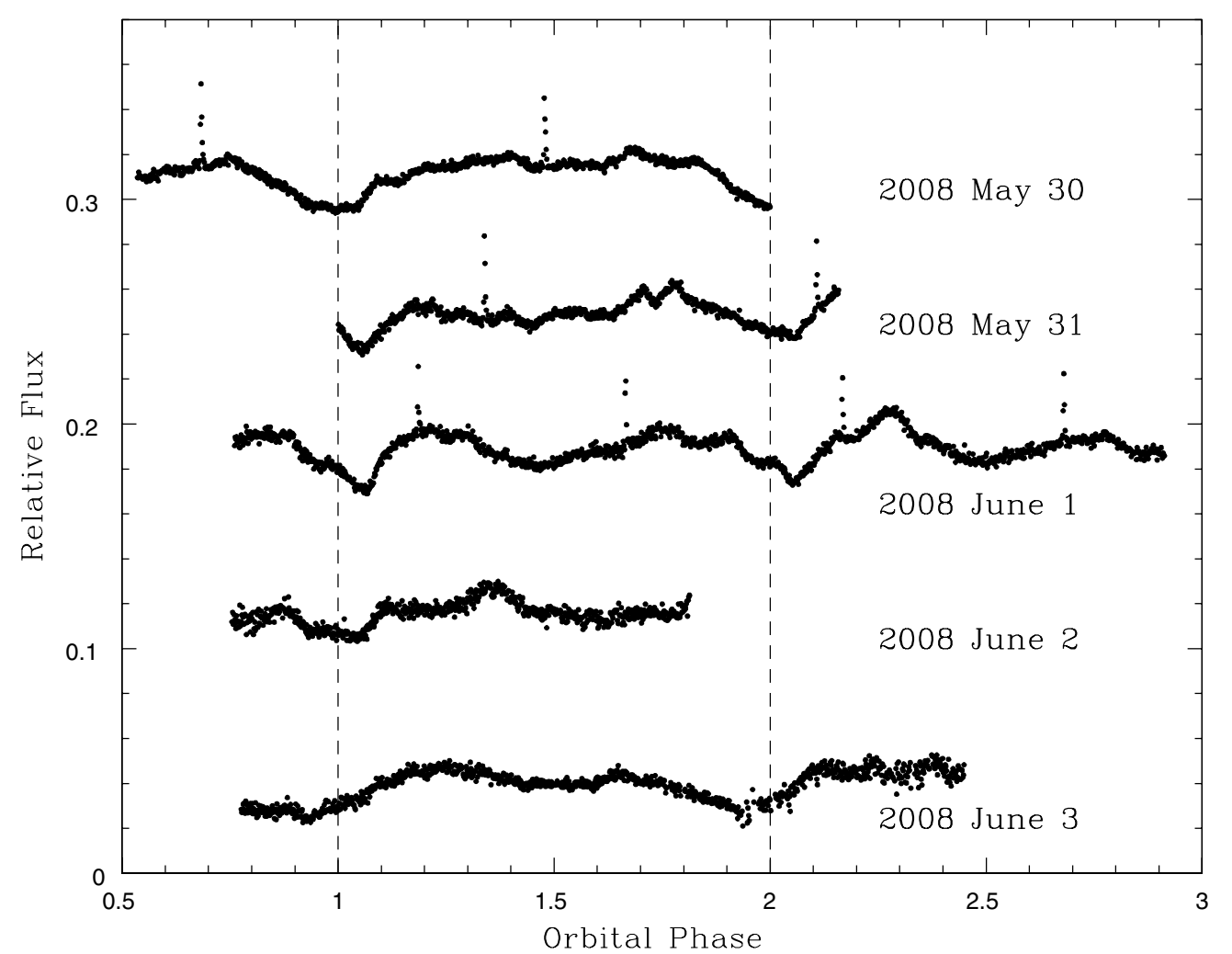

Figure 2. Optical photometry of UW CrB on five successive nights in 2008. The bottom light curve is plotted to scale. The others have been successively offset by 0.07 in relative flux for clarity. The orbital phases are calculated from Equation (1). The shape, depth, and orbital phase of the eclipses vary from night to night. Much of the variation is not random but recurs with a quasi-period of 5.5 days, which is interpreted as the precession period of the elliptical accretion disk.

not strictly periodic but does maintain coherence for $\sim 80$ days. The morphology of the modulation varies greatly from night to night. Muhli et al. (2004) suggested that the morphological variations are not random but repeat at a period near 5.5 days, a result confirmed by Mason et al. (2008) and Hakala et al. (2009). Figure 2 shows the optical light curve of UW CrB on successive nights from May 30 through 2008 June 3, covering most of the 5.5 day period. Additional examples of the 5.5 day cycle are given in similar figures in Hynes et al. (2004) and Mason et al. (2008). Mason et al. (2008) showed that the variations in the phase of the eclipses are also not random but repeat at the same 5.5 day period. The three periods are related. The 5.5 day period is the beat period between the 111 minute orbital period and the 112.6 minute period of the non-eclipse modulation.

The morphology of the non-eclipse modulation and the ratio of its period to the orbital period are similar to those of superhumps seen in the light curves of many cataclysmic variables (Patterson 2001). While there is no universal agreement on the physical mechanisms responsible for superhumps, the common ingredient in all superhump models is a precessing, nonaxisymmetric accretion disk (Haswell et al. 2001; Smith et al. 2007; Smak 2010). Mason et al. (2008) invoked a precessing elliptical accretion disk to explain the variations of eclipse depths and orbital phases of eclipse minima in UW $\mathrm{CrB}$. They were able to match the observations with an accretion disk that has an eccentricity near 0.5 , extends almost to the tidal truncation radius, and precesses with a period of 5.5 days. The approximate geometry of the disk is shown in Figure 3. Even if the optical light curves can be explained mostly by invoking a precessing, elliptical accretion disk, the orbital X-ray modulation implies out of the orbital plane structure, perhaps a non-axisymmetric flared or warped accretion disk (Hakala et al. 2009) or a non-

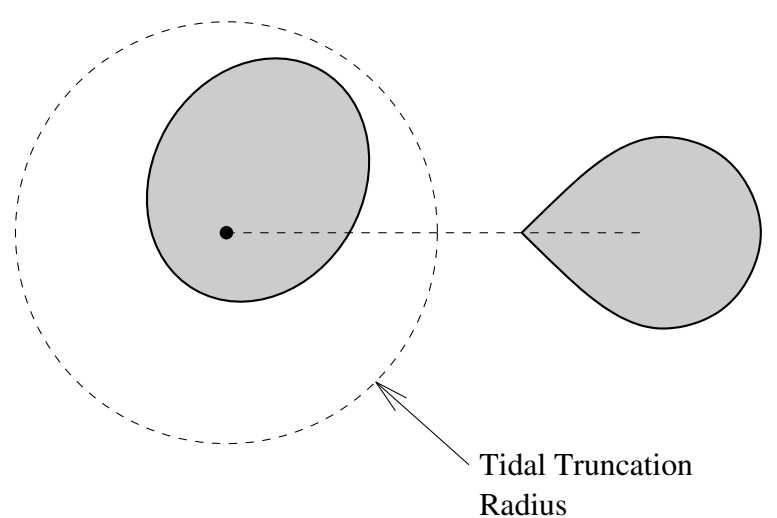

Figure 3. Schematic diagram showing the geometry of the elliptical accretion disk in UW CrB. The disk has an eccentricity of $\sim 0.5$ and extends almost to the tidal truncation radius, shown by the dashed circle. The disk precesses in the prograde direction with a sidereal period of 5.5 days. The eclipse is deepest when the long axis of the disk points at the secondary star, and the phase of mid-eclipse reaches maximum displacement from phase 0 when the long axis points $\pm 60^{\circ}$ away from the secondary.

axisymmetric disk wind similar to that observed in another ADC source X1822-371 (Hellier \& Mason 1989; Bayless et al. 2010).

The fourth kind of variability is the optical counterparts of the type I X-ray bursts (Hynes et al. 2004; Muhli et al. 2004). A typical burst occurred just after the end of the eclipse shown in Figure 1, rising to maximum in less than $10 \mathrm{~s}$ and then fading over the next $60 \mathrm{~s}$. The $e$-folding decay times of the bursts are typically 10-30 s, and both the peak flux and the fluence show surprisingly little variation; see Hynes et al. (2004) and Hakala et al. (2009). The fluence was constant to within $10 \%$ in the 


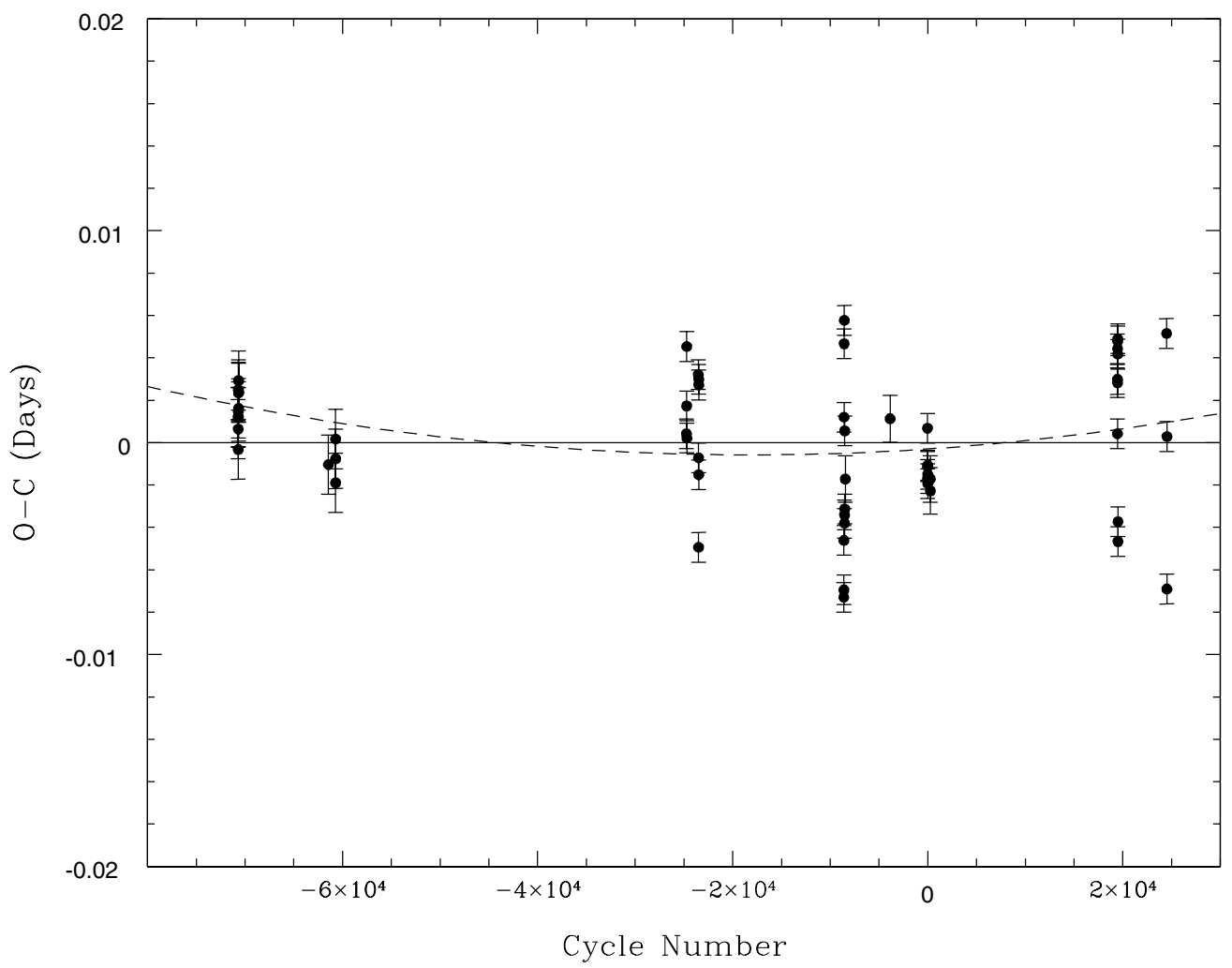

Figure 4. Top panel: $O-C$ diagram for the eclipses of $\mathrm{UW} \mathrm{CrB}$ with respect to the best-fit linear ephemeris given by Equation (1). The error bars correspond to the errors in measuring the times of mid-eclipse. The measurement errors are much less than the displacements introduced by the precession of the elliptical disk. The dashed line is the best-fit quadratic ephemeris. The quadratic term is not statistically significant.

bursts analyzed by Hynes et al. (2004), and the peak flux varied by $\pm 45 \%$ in the bursts observed by Hakala et al. (2009).

\subsection{The Eclipse Ephemeris}

Measurement of the eclipse ephemeris for $\mathrm{UW} \mathrm{CrB}$ is challenging because of its precessing asymmetric disk. The asymmetry introduces a large scatter in the orbital phases of mideclipse, and the orbital phases do not scatter randomly but shift systematically as the disk precesses. To improve the ephemeris, one must observe many eclipses and fully sample the 5.5 day precession period, ideally over five or more successive nights.

Our new data yielded 12 eclipse times. The eclipses cover the 5.5 day superhump beat cycle twice, in early 2008 May/June and again in 2009 June, and are ideal for refining the eclipse ephemeris. We also re-analyzed the extensive photometry discussed by Hakala et al. (2009), extracting another 20 eclipse times. The 32 new eclipse times are listed in Table 2. We combined these times with the 24 eclipse times listed in Table 2 in Mason et al. (2008) for a total of 56 eclipses spanning 20 years. A weighted least-squares fit of a linear ephemeris to the 56 eclipse times yielded

$$
T=\text { HJD 2453118.8380(5) + 0.07706720(2)E, }
$$

where $E$ is the integer eclipse number. The $O-C$ (ObservedCalculated) diagram, with respect to this ephemeris, is shown in Figure 4. The error bars in the figure correspond to the measurement errors on the times of the eclipses. The scatter of the observed eclipse times about this ephemeris is much larger than the measurement error because of the phase displacements introduced by the precessing accretion disk. The best-fit quadratic ephemeris is shown as the dashed line in the figure.
Table 2 Additional Times of Eclipses in UW CrB

\begin{tabular}{lccc}
\hline \hline $\begin{array}{l}\text { Eclipse } \\
\text { Number }\end{array}$ & $\begin{array}{c}\text { Eclipse Time }^{\mathrm{a}} \\
\text { HJD-2,450,000.0 }\end{array}$ & $\begin{array}{c}\text { Eclipse } \\
\text { Number }\end{array}$ & $\begin{array}{c}\text { Eclipse Time }^{\mathrm{a}} \\
\text { HJD-2,450,000.0 }\end{array}$ \\
\hline-24733 & 1212.7353 & -8530 & 2461.4510 \\
-24720 & 1213.7385 & -8529 & 2461.5284 \\
-24707 & 1214.7432 & -8517 & 2462.4535 \\
-24694 & 1215.7408 & -8516 & 2462.5343 \\
-23528 & 1305.6041 & 19449 & 4617.7184 \\
-23501 & 1307.6768 & 19450 & 4617.7980 \\
-23489 & 1308.6058 & 19461 & 4618.6472 \\
-23488 & 1308.6821 & 19462 & 4618.7227 \\
-23476 & 1309.6111 & 19474 & 4619.6496 \\
-23475 & 1309.6884 & 19475 & 4619.7265 \\
-8608 & 2455.4362 & 19487 & 4620.6507 \\
-8595 & 2456.4385 & 19500 & 4621.6438 \\
-8594 & 2456.5179 & 19487 & 4621.7218 \\
-8581 & 2457.5256 & 24484 & 5005.7565 \\
-8569 & 2458.4538 & 24509 & 5007.6711 \\
-8557 & 2459.3797 & 24522 & 5008.6802
\end{tabular}

Note. ${ }^{a}$ Eclipse times are accurate to \pm 0.0007 days.

The quadratic term is not statistically significant. The upper limit to the rate of change of the orbital period is $|\dot{P}|<4.2 \times 10^{-11}$ (unitless) with $90 \%$ confidence.

It is not possible to convert the upper limit on $|\dot{P}|$ to an upper limit on the rate of mass transfer without further assumptions. Dimensional analysis suggests $|\dot{m}| / m \approx|\dot{P}| / P$ where $m$ is the mass scale of the system. Adopting $m=1 M_{\odot}$, we find $|\dot{m}|<2 \times 10^{-7} M_{\odot} \mathrm{yr}^{-1}$. If one is willing to assume that the secondary star is a main-sequence star and follows 


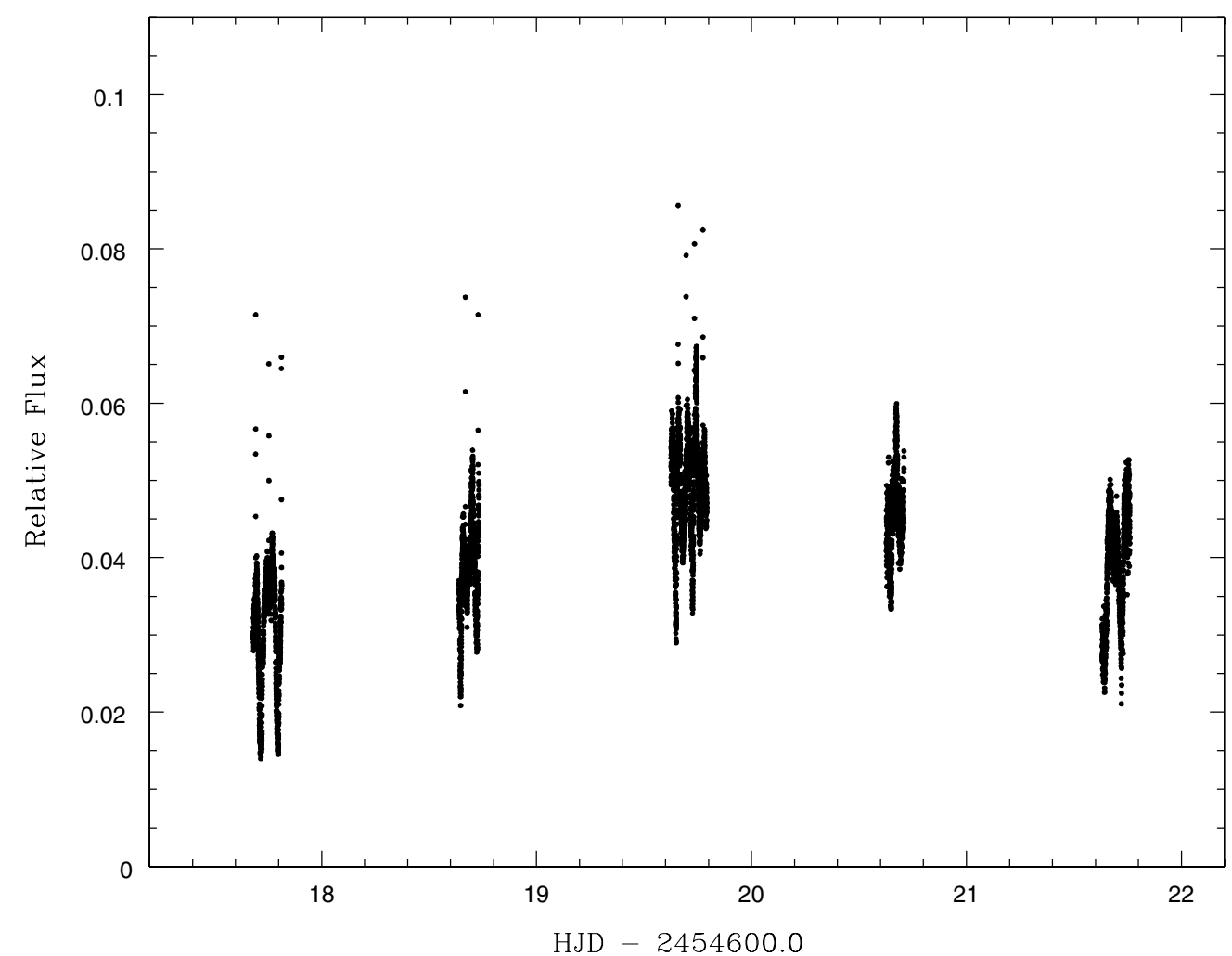

Figure 5. Optical photometry of UW CrB on five successive nights in 2008. The data are the same as that plotted in Figure 2 but the light curves have been plotted against time instead of orbital phase. The flux from UW CrB increased by $\sim 50 \%$ from the first to the third night and then faded over the next two nights. The bursts occurred only on the first three nights.

the empirical mass-radius relation for the secondary stars of cataclysmic variables, then $m_{2}=0.032 P^{2.38}$, where $m_{2}$ is the mass of the secondary star in solar masses and $P$ is the orbital period in hours (Patterson et al. 2005). This yields $\dot{m}_{2} / m_{2}=2.38 \dot{P} / P$. For UW CrB we find $m_{2}=0.14 M_{\odot}$ and $\left|\dot{m}_{2}\right|<6.6 \times 10^{-8} M_{\odot} \mathrm{yr}^{-1}$. Since UW $\mathrm{CrB}$ is an ADC system, it is likely that some mass lost by the secondary star leaves the system altogether and does not wind up on the NS (Bayless et al. 2010).

\subsection{The Optical Bursts}

There were nine optical bursts in our new photometry, all of which are shown in Figure 2. The times of the bursts are listed as the final nine entries in Table 3 along with orbital phases calculated from the new ephemeris. We have also measured the times of bursts in the data discussed in Hynes et al. (2004) and Mason et al. (2008) and have added these to the table. Finally, we have added the 11 burst times listed in Hakala et al. (2009), for a total of 33 bursts.

The burst frequency is far from constant. In Figure 5, the five successive light curves of UW CrB from 2008 May/June have been replotted against time instead of orbital phase. The bursts occurred only on the first three nights. There were no bursts at all during the $13 \mathrm{hr}$ of data obtained on the six successive nights from 2009 June 23 to 28 . Within single nights the interval between bursts is roughly constant-the intervals were 53.4 minutes, 55.6 minutes, and 56.9 minutes for the four bursts on 2008 June 1-but the mean interval between bursts decreased from 86 minutes on the first nights to 55 minutes on the third night. UW CrB brightened by $~ 50 \%$ from the first to the third night and then faded over the next two nights, leading us to search for correlations between burst occurrence
Table 3

Times and Orbital Phases of Optical Bursts from UW CrB

\begin{tabular}{lccccc}
\hline \hline HJD-2,400,000.0 & $\begin{array}{c}\text { Orbital } \\
\text { Phase }^{\mathrm{a}}\end{array}$ & $\begin{array}{c}\text { Relative } \\
\text { Fluence }\end{array}$ & HJD-2,400,000.0 & $\begin{array}{c}\text { Orbital } \\
\text { Phase }^{\mathrm{a}}\end{array}$ & $\begin{array}{c}\text { Relative } \\
\text { Fluence }\end{array}$ \\
\hline 51213.7470 & 0.132 & 0.82 & 53118.8507 & 0.165 & 0.92 \\
51214.6890 & 0.355 & 0.92 & 53137.7410 & 0.279 & 0.37 \\
51215.7670 & 0.343 & 0.76 & 53138.9008 & 0.329 & 0.36 \\
51304.6140 & 0.194 & 0.58 & 53139.8254 & 0.326 & 0.32 \\
51304.6980 & 0.284 & 0.71 & 53142.8630 & 0.741 & 0.39 \\
51307.6130 & 0.108 & 0.88 & 53171.6812 & 0.677 & 0.21 \\
51308.5680 & 0.500 & 0.41 & 53171.8010 & 0.231 & 0.24 \\
51309.6370 & 0.371 & 0.38 & 54617.6935 & 0.682 & 0.85 \\
52439.7112 & 0.862 & 0.89 & 54617.7548 & 0.478 & 0.84 \\
52461.4380 & 0.782 & 0.32 & 54617.8133 & 0.237 & 0.81 \\
52462.4750 & 0.238 & 0.89 & 54618.6689 & 0.339 & 0.80 \\
52468.7229 & 0.309 & 0.28 & 54618.7281 & 0.107 & 0.61 \\
52492.4220 & 0.821 & 0.46 & 54619.6589 & 0.185 & 0.58 \\
53111.9086 & 0.086 & 0.69 & 54619.6960 & 0.666 & 0.59 \\
53111.9846 & 0.072 & 0.31 & 54619.7346 & 0.167 & 0.58 \\
53116.8760 & 0.542 & 0.44 & 54619.7741 & 0.680 & 0.71 \\
53117.9697 & 0.733 & 0.58 & & & \\
\hline
\end{tabular}

Note. ${ }^{\text {a }}$ Phase zero is the time of mid-eclipse given by Equation (1).

and mean brightness. We were unable to find any convincing correlation. This is perhaps not surprising as theory suggests that burst properties should correlate more strongly with X-ray luminosity than with visual brightness (Strohmayer \& Bildsten 2006). We also search for correlations with 5.5 day superhump beat cycle but, again, did not find anything convincing.

Figure 6 shows the distribution of the 33 optical bursts in orbital phase. Each line in the figure represents one burst and is angled toward the orbital phase at which the burst occurred. 


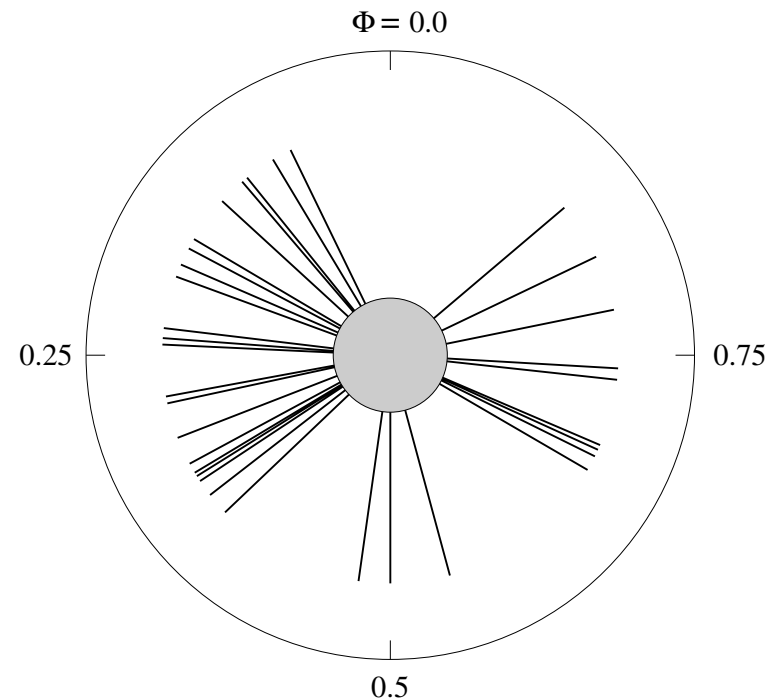

Figure 6. Distribution of the 33 optical bursts from UW $\mathrm{CrB}$ in orbital phase. Each line represents one burst and is angled toward the orbital phase at which the burst occurred. No bursts occurred in a gap centered on phase 0.967 with width $\Delta \phi=0.210$.

No bursts occurred in a gap centered at phase 0.967 with width $\Delta \phi=0.210 .^{7}$ A Monte Carlo simulation showed that the probability of finding a gap this wide at an arbitrary orbital phase is $\sim 1.3 \%$ if the bursts are randomly distributed in phase. However, the probability that no bursts fall within a phase gap this wide centered on a pre-specified range of phases, in this case within \pm 0.04 of phase 0.0 , is much smaller, $0.08 \times 1.3 \approx 0.1 \%$.

7 The differences between our results and those of Hakala et al. (2009) on this point are due entirely to our use of Equation (1) to calculate orbital phases.
A visual inspection of the light curves revealed a broader result: no bursts were observed during any eclipse, no matter what its width.

Visual inspection of the bursts in Figure 2 shows that they reached the same peak flux to within $\pm 10 \%$ and that lowamplitude bursts are notably absent. However, since the bursts are unresolved in the optical data, fluence is a more meaningful quantity than peak flux. Table 3 gives the fluences for the 33 bursts. All the light curves of UW CrB measured at McDonald Observatory were measured through the same broadband filter and referenced to the same comparison star, and are on the same (albeit uncalibrated) flux scale. The fluences of the 11 bursts observed by Hakala et al. (2009) are given in the fourth column of their Table 3 (the column is mislabeled). While these fluences were also measured through a broadband filter, they are normalized to a flux of 1.0 before and after the burst. The fluences have been scaled to the typical mean flux of UW CrB in the McDonald data.

Figure 7 shows the relative fluences of the bursts as a function of orbital phase. In principle, type I X-ray bursts can reprocess to optical flux from both the accretion disk and the secondary star. Numerical simulations by O'Brien et al. (2002) and Hynes (2008) show that the optical fluence of bursts reprocessed on the secondary star is a strong function of orbital phase because only one side of the secondary is heated. For systems with orbital inclinations high enough to show eclipses, the reprocessed bursts should nearly disappear for $\sim 1 / 4$ of the orbit centered on the eclipse. In contrast, the optical bursts from UW CrB are seen at full fluence until they disappear altogether inside the gap at phase zero. Most of the flux in the optical bursts must, therefore, be coming from the accretion disk, not the secondary star. We suggest that no optical bursts are observed within the gap because at those phases the secondary star obscures the

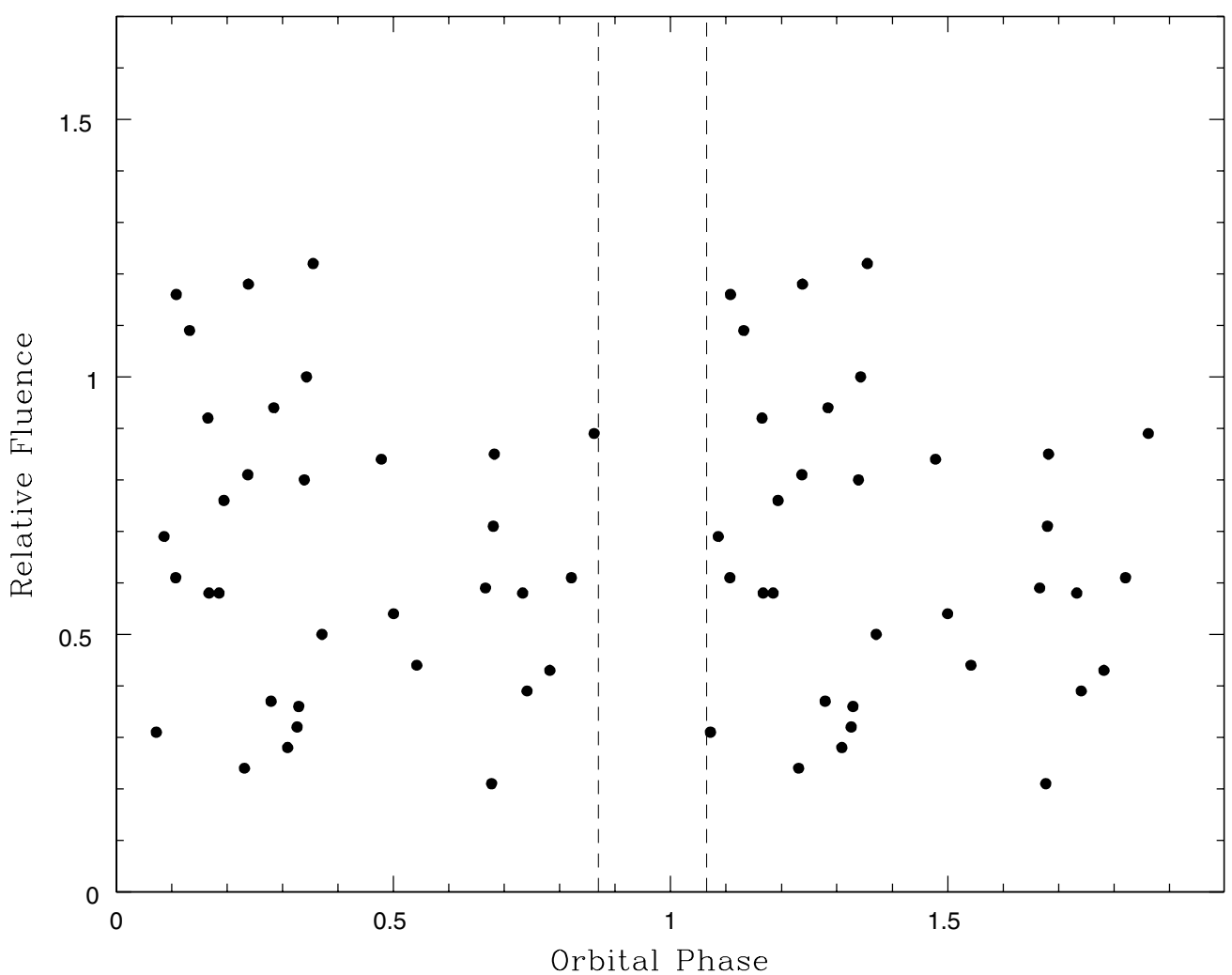

Figure 7. Relative fluence of the 33 optical bursts from $\mathrm{UW} \mathrm{CrB}$ as a function of orbital phase. No bursts occurred in a gap centered on phase 0.967 with width $\Delta \phi=0.210$. The relative fluence in the bursts is roughly the same at all orbital phases where they are seen; there is no progressive diminution toward phase 0 . 


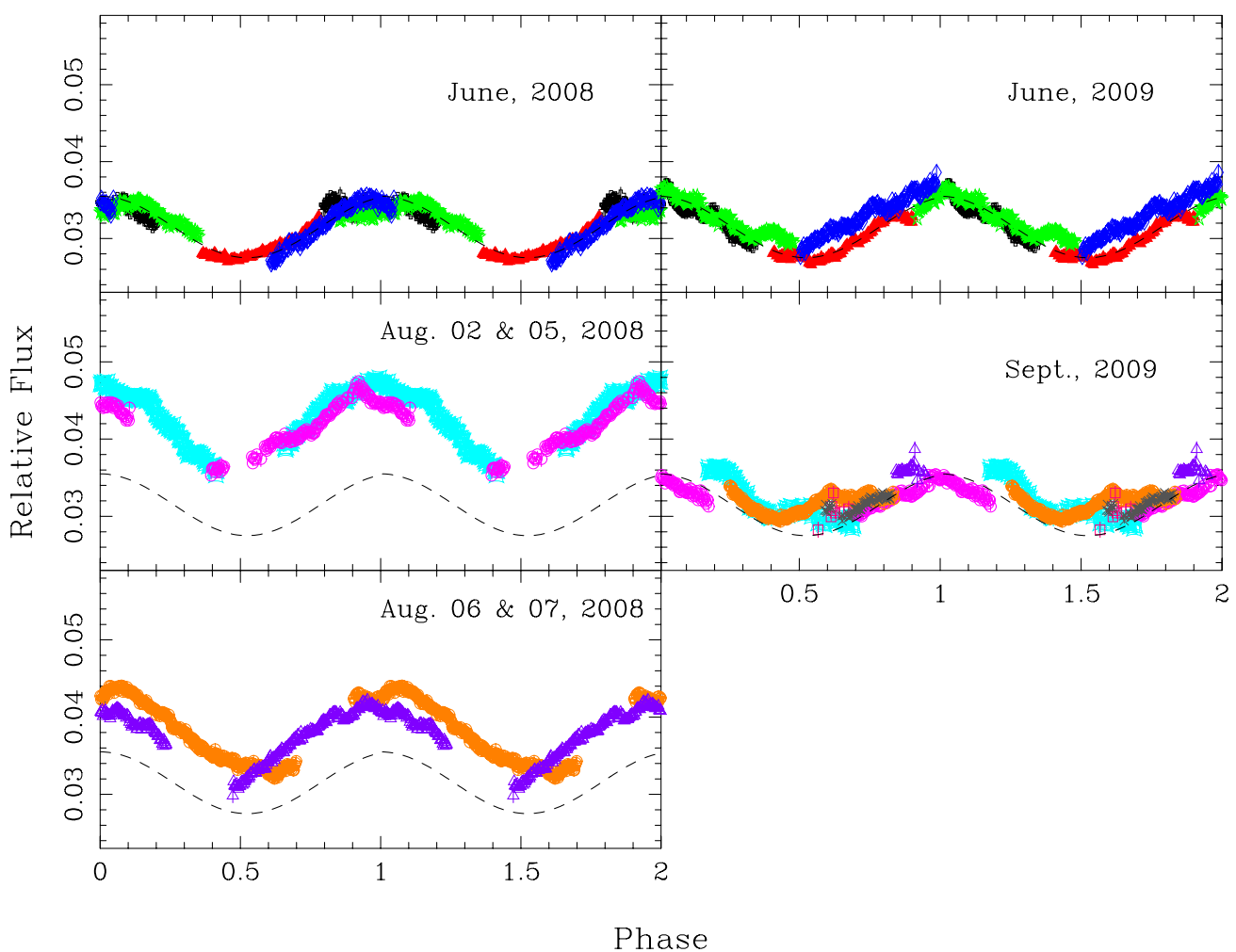

Figure 8. Light curves of V1408 Aql in 2008 and 2009. The light curves for individual months have been folded in phase according to the ephemeris in Equation (2). The error bars are based on photon counting noise. The dashed line is a sine curve fitted to the 2008 June data and then reproduced in the other panels. The orbital modulation is clearly present in the new data although rapid flux variations masked the modulation somewhat in 2009 September.

(A color version of this figure is available in the online journal.)

parts of the disk where the X-ray bursts are reprocessed to optical flux. Hakala et al. (2009) have noted that the nearly constant amplitude of the optical bursts also places limits on the asymmetry of the accretion disk, although the limits depend strongly on the disk model.

Because the $10 \mathrm{~s}$ exposures of our new photometry are twice those of the best photometry in Hynes et al. (2004), we have nothing useful to add to the discussion about the burst profiles in that paper. We do note that we see no long bursts that would contradict the short length and $e$-folding decay times measured by Hynes et al. (2004) and Hakala et al. (2009).

\section{V1408 AQL}

\subsection{Background}

4U $1957+115$ is a persistent $X$-ray source with a luminosity between $\sim 10^{36}$ and $\sim 10^{38} \mathrm{erg} \mathrm{s}^{-1}$ (Russell et al. 2010). Its $\mathrm{X}$-ray flux varies on all timescales from a few tenths of a second to years (Wijnands et al. 2002; Russell et al. 2010); however, its RXTE/ASM X-ray light curve shows no eclipses or other variability at the orbital period, or any recurring quasiperiods that might be attributable to disk warping, superhumps, precession, or other disk asymmetries (Bayless et al. 2011). Its X-ray spectrum is unusually soft, placing 4U 1957+115 in the region of the X-ray color-color diagram occupied by black hole binaries, leading to the suspicion that $4 U 1957+115$ harbors a black hole (White \& Marshall 1984; Schulz et al. 1989; McClintock \& Remillard 2003). Models in which the accretion disk is accreting onto a rapidly spinning black hole fit the observed X-ray spectrum well (Nowak et al. 2008, 2012). As noted by Nowak et al. (2012), however, the mass, orbital inclination, and distance to $4 \mathrm{U} 1957+115$ are unknown, and the evidence that it contains a black hole is indirect. Bayless et al. (2011) also note that there is some circumstantial evidence that the compact object is a NS. If 4 U 1957+115 does contain a black hole, it would be the only known black hole LMXB in the Galaxy that does not show transient X-ray outbursts.

The light curve of V1408 Aql, the optical counterpart to $4 \mathrm{U} 1957+115$, has a $9.33 \mathrm{hr}$ modulation, presumed to be the orbital period. Thorstensen (1987) and Bayless et al. (2011) both found that the mean orbital light curve was nearly sinusoidal with an amplitude of $23 \%$. However, the light curve also varies randomly by up to $0.05 \mathrm{mag}$ on timescales of a half-hour (Bayless et al. 2011) and up to $0.8 \mathrm{mag}$ on timescales of days (Russell et al. 2010), and these variations tend to obscure the orbital modulation. Thorstensen (1987) needed $\sim 48 \mathrm{hr}$ of photometry to extract a reliable mean light curve and Bayless et al. (2011) needed $\sim 51 \mathrm{hr}$. Orbital light curves constructed from short or sparsely sampled data sets can differ substantially from the true mean orbital light curve. Thus, Hakala et al. (1999) obtained two nights of UBVRI photometry, covering a single orbital cycle in total, and concluded that the light curve was not sinusoidal; Russell et al. (2010) were unable to detect the orbital modulation at all in their sparsely sampled light curves. Because of this, the orbital period has not been easy to refine and is still not known well enough to bridge the gap in data between successive years.

Thorstensen (1987) attributed the orbital modulation at optical wavelengths to the varying aspect of the X-ray-heated face of the secondary star, and Bayless et al. (2011) showed that this model gives an excellent quantitative fit to the optical light curve. This model leaves the orbital inclination nearly 


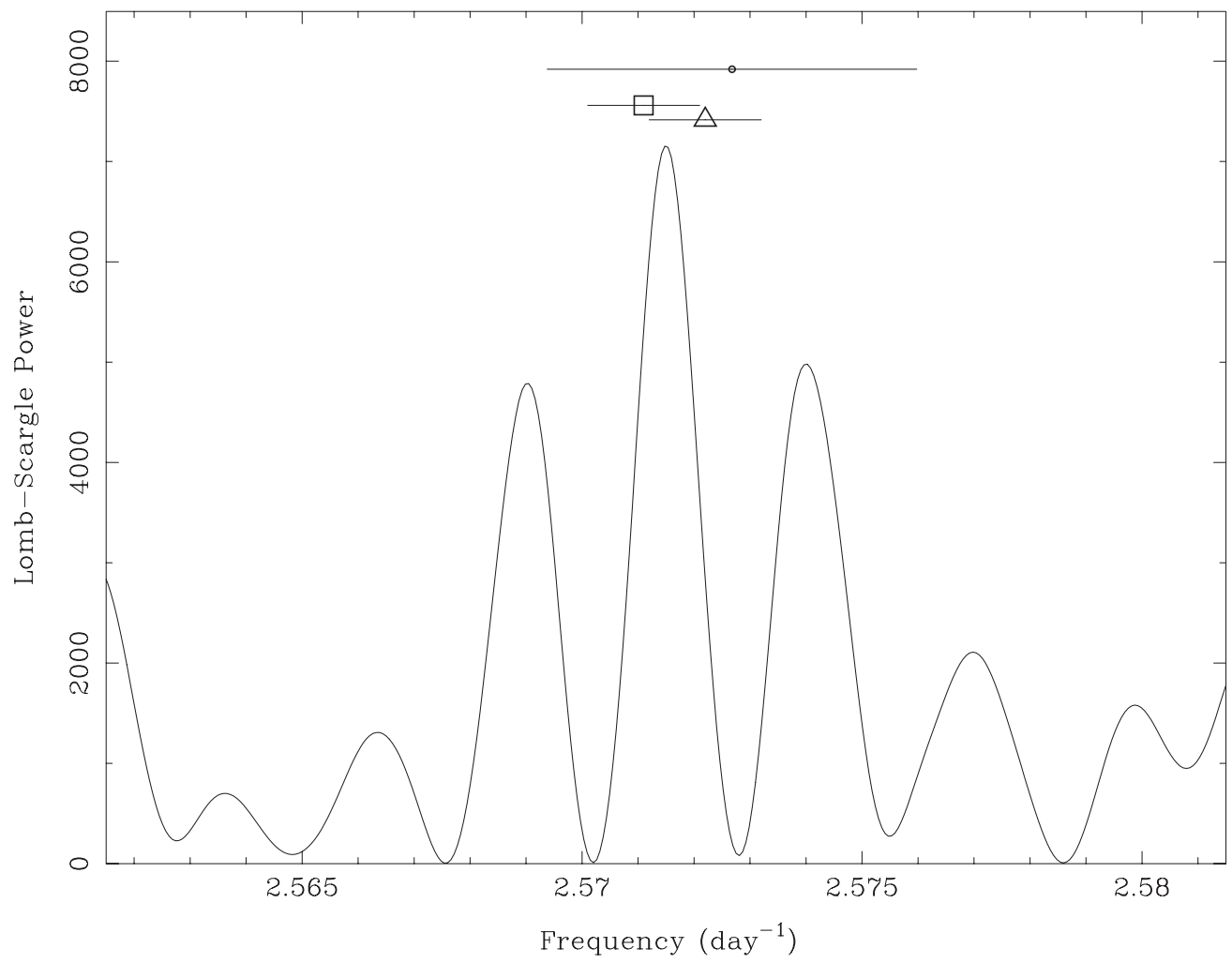

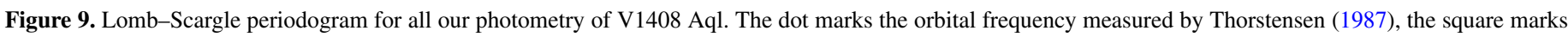

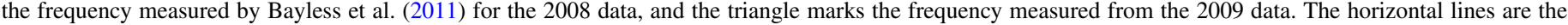
error bars on the frequencies. The side lobes are one-year aliases of the orbital frequency.

unconstrained with permitted inclinations of $\sim 20^{\circ}<i<70^{\circ}$. The model mildly favors a NS primary as it is easier to produce the $23 \%$ amplitude if the mass ratio is high. At the lower mass ratios (higher primary mass) of black hole systems the secondary star subtends a smaller solid angle as seen from the compact star, and also suffers greater shadowing by the accretion disk. As a consequence, the range of model parameters for which the secondary star is heated to a high enough temperature to reproduce the amplitude of the orbital light curve is greatly restricted.

\subsection{The Improved Ephemeris}

The new photometry of V1408 Aql is shown in Figure 8 along with the photometry obtained in 2008 by Bayless et al. (2011). The dashed curve is a sine curve fitted to the 2008 June data and then reproduced in the other panels. The orbital modulation is obvious in the new data although rapid flux variations masked the modulation somewhat in 2009 September.

To refine the orbital ephemeris, we first calculated the Lomb-Scargle (L-S) periodogram of the 2009 photometry alone (Lomb 1976; Scargle 1982). As expected, the periodogram had a series of evenly spaced peaks at three month aliases introduced by the three month gap in the data. Several of the peaks reached nearly the same significance, of which one was at $2.571 \mathrm{day}^{-1}$, in good agreement with the frequency found by Bayless et al. (2011). Harmonics of this frequency were absent from the periodogram, so the mean orbital light curve was not measurably different from a sine curve. A least-squares fit of a sine curve to the data yielded an amplitude near $23 \%$ in June and about half that in September for a mean amplitude of $18 \%$. Thus, the orbital light curve had the same period and mean shape in 2009 as in 1984 and 2008, but its mean amplitude was somewhat lower.

We then calculated the L-S periodogram for the 2008 and 2009 data together. The mean optical flux from V1408 Aql can shift over the course of a few days and these shifts were particularly large in our 2008 data. In preparation for calculating the L-S periodogram for the entire data set, we normalized all the 2008 and 2009 light curves to the median flux in 2008 June. The L-S periodogram of the conjoined 2008 and 2009 data is shown in Figure 9. It has a single high peak at a frequency of $2.5715 \pm 0.0006$ day $^{-1}$ with side lobes at one-year aliases. The frequencies determined by Thorstensen (1987), by Bayless et al. (2011) from the 2008 data, and by us from the 2009 data are also shown in the figure and uniquely pick out the high peak. Having identified the correct one-year alias, we fit a sine curve to our photometry by least squares to obtain the improved ephemeris:

$$
T=\text { HJD 2454621.829(4) }+0.388879(5) E,
$$

where $T$ is the time of maximum flux. The relatively large error on the time at which $E=0$ results from the distortions in the light curve introduced by flickering and changes in the mean brightness. The light curves shown in Figure 8 have been folded against this ephemeris. Since our data have just a one-year baseline, we cannot give a meaningful upper limit to the rate of change of the orbital period and cannot bridge the 23 year gap between our data and that of Thorstensen (1987).

\section{CONCLUSIONS}

We obtained 12 new times of eclipse for UW CrB. Combining these times with 44 eclipse times extracted from previously 
published data, we have derived an improved eclipse ephemeris, which is given by Equation (1). The scatter of the observed eclipse times about this ephemeris is much larger than the measurement errors because of the phase displacements introduced by the precessing elliptical accretion disk. The upper limit to the rate of change of the orbital period is $|\dot{P}|<4.2 \times 10^{-11}$ (unitless). If the secondary star is a main-sequence star and follows the empirical mass-radius relation for the secondary stars of cataclysmic variables, the upper limit to the rate of change of its mass is $\left|\dot{m}_{2}\right|<6.6 \times 10^{-8} M_{\odot} \mathrm{yr}^{-1}$.

Table 3 lists the times, orbital phases, and relative fluences of 33 optical bursts from UW CrB. No bursts were observed in a gap centered on orbital phase $\phi=0.97$ with width $\Delta \phi=0.21$. If the bursts were randomly distributed in orbital phase, the probability that no bursts would fall within a phase gap that is this wide and centered within \pm 0.04 of phase 0.0 is $\sim 0.1 \%$. No bursts were observed during any eclipse. The relative fluence in the bursts is roughly the same at all orbital phases where they are seen; there is no progressive diminution toward phase 0 . We infer that the optical flux in the bursts comes primarily from the accretion disk, not from the secondary star, and that the lack of bursts near phase zero is caused by eclipses of those parts of the disk where the X-ray flux in the bursts is reprocessed to optical flux.

The mean orbital light curve of V1408 Aql was a sine curve in 2009 as it was in 1984 and 2008, but its mean amplitude was slightly lower, $18 \%$ against $23 \%$ in the earlier data. The model proposed by Bayless et al. (2011) in which the orbital modulation is caused by the varying aspect of the heated face of the secondary star continues, therefore, to give an excellent quantitative fit to the light curve. The optical light curve also continues to show additional variations and distortions on timescales from minutes to months. The new photometry of V1408 Aql allows an unambiguous count of the orbital periods that elapsed over the gap between annual observing seasons. Equipped with the cycle count we have derived a greatly improved orbital ephemeris, given by Equation (2).

We thank the anonymous referee for suggestions that resulted in significant improvements to this paper. This research was supported in part by the NSF grant No. 0958783.

\section{REFERENCES}

Bayless, A. J., Robinson, E. L., Hynes, R. I., Ashcraft, T. A., \& Cornell, M. E. 2010, ApJ, 709, 251

Bayless, A. J., Robinson, E. L., Mason, P. A., \& Robertson, P. 2011, ApJ, 730, 43

Gioia, I. M., Maccacaro, T., Schild, R. E., et al. 1990, ApJS, 72, 567

Hakala, P., Hjalmarsdotter, L., Hannikainen, D. C., \& Muhli, P. 2009, MNRAS, 394, 892

Hakala, P., Ramsay, G., Muhli, P., et al. 2005, MNRAS, 356, 1133

Hakala, P. J., Muhli, P., \& Dubus, G. 1999, MNRAS, 306, 701

Haswell, C. A., King, A. R., Murray, J. R., \& Charles, P. A. 2001, MNRAS, 321,475

Hellier, C., \& Mason, K. O. 1989, MNRAS, 239, 715

Hynes, R. I. 2008, in AIP Conf. Proc. 984, High Time Resolution Astrophysics: The Universe at Sub-Second Timescales, ed. D. Phelan, O. Ryan, \& A Shearer (Melville, NY: AIP), 3

Hynes, R. I., Robinson, E. L., \& Jeffery, E. 2004, ApJ, 608, 101

Jonker, P. G., van der Klis, M., Kouveliotou, C., et al. 2003, MNRAS, 346, 684

Lomb, N. R. 1976, Ap\&SS, 39, 447

Mason, P. A., Robinson, E. L., Gray, C. L., \& Hynes, R. I. 2008, ApJ, 685, 428

McClintock, J. E., \& Remillard, R. A. 2003, in Compact Stellar X-Ray Sources, ed. W. H. G. Lewin \& M. van der Klis (Cambridge: Cambridge Univ. Press), 157

Morris, S. L., Liebert, J., Stocke, J. T., et al. 1990, ApJ, 365, 686

Muhli, P., Hakala, P. J., Hjalmarsdotter, L., Hannikainen, D. C., \& Schultz, J. 2004, RevMexAA, 20, 211

Mukai, K., Smale, A. P., Stahle, C. K., Schlegel, E. M., \& Wijnands, R. 2001, ApJ, 561, 938

Nather, R. E., \& Mukadam, A. S. 2004, ApJ, 605, 846

Nowak, M. A., Juett, A., Homan, J., et al. 2008, ApJ, 689, 1199

Nowak, M. A., Wilms, J., Pottschmidt, K., et al. 2012, ApJ, 744, 107

O’Brien, K. O., Horne, K., Hynes, R. I., et al. 2002, MNRAS, 334, 426

Patterson, J. 2001, PASP, 113, 736

Patterson, J., Kemp, J., Harvey, D. A., et al. 2005, PASP, 117, 1204

Russell, D. M., Louis, F., Roche, P., Clark, J. S., \& Fender, R. P. 2010, MNRAS, 402, 2671

Scargle, J. D. 1982, ApJ, 263, 835

Schulz, N. S., Hasinger, G., \& Trümper, J. 1989, A\&A, 225, 48

Smak, J. 2010, Acta Astron., 60, 1

Smith, A. J., Haswell, C. A., Murray, J. R., Truss, M. R., \& Foulkes, S. B. 2007, MNRAS, 378, 785

Strohmayer, T., \& Bildsten, L. 2006, in Compact Stellar X-Ray Sources, ed. W. H. G. Lewin \& M. van der Klis (Cambridge: Cambridge Univ. Press), 113

Thorstensen, J. R. 1987, ApJ, 312, 739

Warner, B. 1995, Cataclysmic Variable Stars (Cambridge: Cambridge Univ. Press)

White, N. E., \& Marshall, F. E. 1984, ApJ, 281, 354

Wijnands, R., Miller, J. M., \& van der Klis, M. 2002, MNRAS, 331, 60 